\title{
Advances and Challenges in Convergent Communication Networks
}

\author{
Homero Toral-Cruz ${ }^{1}$ - Albena D. Mihovska ${ }^{2}$. \\ Piotr $\mathrm{Gaj}^{3} \cdot$ Debiao $\mathrm{He}^{4} \cdot$ Julio Cesar Ramírez-Pacheco ${ }^{6}$. \\ Miroslav Voznak ${ }^{5} \cdot$ Izabella Lokshina $^{7}$
}

Published online: 11 September 2017

(C) Springer Science+Business Media, LLC 2017

Welcome to this special issue of Wireless Personal Communications on Advances and Challenges in Convergent Communication Networks. The main purpose of this special issue is to present new progresses and challenges in convergent networks.

Communication networks play an important role in our daily life because they allow communicating and sharing contents between heterogeneous nodes around the globe. The emergence of multiple network architectures and emerging technologies have resulted in

Homero Toral-Cruz

htoral@uqroo.edu.mx; homerotoralcruz@gmail.com

Albena D. Mihovska

albena@es.aau.dk

Piotr Gaj

piotr.gaj@polsl.pl

Debiao He

hedebiao@163.com

Julio Cesar Ramírez-Pacheco

jramirez@ucaribe.edu.mx

Miroslav Voznak

miroslav.voznak@vsb.cz

Izabella Lokshina

Izabella.Lokshina@oneonta.edu

1 Department of Sciences and Engineering, University of Quintana Roo, Boulevard Bahía s/n Esq. Ignacio Comonfort, Col. del Bosque, 77019 Chetumal, Quintana Roo, Mexico

2 Center for TeleInFrastructur, Aalborg University, Fredrik BajersVej 7A, Room C1-113, P.O. Box 159, 9220 Aalborg, Denmark

3 Faculty of Automatic Control, Electronics and Computer Science, Institute of Informatics, Silesian University of Technology, Ul. Akademicka 16, 44-100 Gliwice, Poland

4 State Key Lab of Software Engineering, Computer School, Wuhan University, Wuchang, Wuhan 430072, Hubei, China 
new applications and services over a heterogeneous network. This heterogeneous network has undergone significant challenges in recent years, such as the evolution to a converged network with the capability to support multiple services, while maintaining a satisfactory level of QoE/QoS, security, efficiency and trust.

The special issue on Advances and Challenges in Convergent Communication Networks is composed by twenty nine high quality original papers covering theoretical and practical aspects on this emerging topic in order to provide novel ideas and directions for the relevant scientific industrial and academic community. We have selected a set of best papers from: a) 15th International Conference Knowledge in Telecommunication Technologies and Optics (KTTO 2015); b) 22th International Conference on Computer Networks (CN 2015); and c) the academic community around the globe (for researchers who could not participate in the above mentioned conferences). All conference papers have been extended and reviewed again by three independent reviewers.

This special issue starts with the paper entitled "PEAL: Power Efficient and Adaptive Latency Hierarchical Routing Protocol for Cluster-Based WSN" by Hidoussi et al., where a novel protocol for cluster-based wireless sensor networks called PEAL (Power Efficient and Adaptive Latency) is proposed. The authors show by means of simulation results that PEAL can extend the network lifetime about $47 \%$ compared to the classic protocol LEACH (Low-Energy Adaptive Clustering Hierarchy) and introduces an acceptable transmission latency compared to the energy conservation gain.

The second paper, entitled "Network Coding Based Converge-cast Scheme in Wireless Sensor Networks" by Xu et al., proposes a Converge-cast Scheme based on data collection Rate Prediction (CSRP) to improve the WSNs' energy efficiency. Furthermore, a novel coding conditions based on Connected Dominating Set (CDS) is proposed to increase the coding opportunity and to solve the collision problems.

The third paper, entitled "MMSMAC: A Multi-mode Medium Access Control Protocol for Wireless Sensor Networks with Latency and Energy-Awareness" by Guerroumi et al., presents a new Medium Access Control (MAC) protocol for Wireless Sensor Networks called MMSMAC, which operates according to the application requirements and traffic load, in three main modes: synchronous, asynchronous, and hybrid. With these characteristics, the proposed protocol, outperforms previous contributions based on CSMA, TDMA and B-MAC protocols.

The fourth paper, entitled "Weighted Hyperbolic DV-Hop Positioning Node Localization Algorithm in WSNs" by Mass-Sanchez et al., presents a performance evaluation of multi-hop localization range-free algorithms used in Wireless Sensor and Actors Networks (WSAN), such as Distance Vector Hop (DV-Hop), Improved DV-Hop (IDVHop), and the Weighted DV Hop (WDV-Hop). In addition, a new localization algorithm, merging WDV Hop, with the weighted hyperbolic localization algorithm (WH) is proposed, which includes weights to the correlation matrix of the estimated distances between the node of interest (NOI) and the reference nodes (RN) in order to improve accuracy and precision.

5 Department of Telecommunication, VSB-Technical University of Ostrava, 17. Listopadu 15/2172, 70833 Ostrava, Czech Republic

6 Department of Basic Sciences and Engineering, DCBeI, University of Caribe, SM-78, Mza-1, Lote1, Esquina Fraccionamiento Tabachines, 77528 Cancún, Mexico

7 Department of Management, Marketing and Information Systems, State University of New York at Oneonta, 108 Ravine Pkwy, Oneonta, NY 13820, USA 
The fifth paper, entitled "A Low Duty Cycle MAC Protocol for Directional Wireless Sensor Networks" by Narin Nur et al., proposes a MAC protocol for Directional Wireless Sensor Networks (DWSNs), termed as DCD-MAC, and based on low duty cycling. The proposed mechanism ensures synchronized transmission-reception between all parentchild pairs. All DCD-MAC nodes schedules the collision free data transmission slots in distributed manner. Each parent allocates slots to its child nodes proportionate to their requirements. Thus, an energy-efficient and highly scalable protocol is developed that allows a sensor node to switch into sleep state whenever it is not scheduled to receive or transmit any data packets.

The sixth paper, entitled "MRL-SCSO: Multi-agent Reinforcement Learning-Based Self-Configuration and Self-Optimization Protocol for Unattended Wireless Sensor Networks" by Renold et al., presents the design and implementation of protocol called MRLSCSO that considered self-configuration and self-optimization for unattended wireless sensor networks (UWSN). Self-configuration is achieved by adaptively activating the neighbors determined by MRL, and the boundary formation is performed using the energyaware convex-hull algorithm to optimize the network performance under heavy load conditions.

The seventh paper, entitled "Self-organizing mobility control in wireless sensor and actor networks based on virtual electrostatic interactions" by Płaczek et al., refers to the findings of the new mobility control method for surveillance applications of wireless sensor and actor networks. The proposed method is based on virtual electrostatic forces and enables effective deployment of actors at the start phase as well as adaptation of actors' placement to variable conditions without the need of any central controller.

The eighth paper, entitled "A Contract-based Model for Multiuser Cooperative Relay in Wireless Communication Networks" by Zhao, studies the relay incentive mechanism between multiple sources and multiple RNs in the wireless cooperative communication networks. Considering the selfish nature of the sources and the RNs, cooperative communication was modeled as a labor market to motivate the RNs to participate in cooperative relay efficiently and credibly. The contract model with relay factor was presented to describe the multiple nodes' contract-relay strategy. Simulation results showed that the optimal contract design scheme could achieve the better cooperative utility of sources compared with equal relay-power and equal relay-factor strategies.

The ninth paper, entitled "Underwater Ad Hoc Wireless Communication for Video Delivery" by Sendra et al., studies the EM wave's behavior at $2.4 \mathrm{GHz}$ when the temperature is varied in underwater freshwater environments. This study was carry out, fixing the water conditions and measuring the maximum distance as a function of several network parameters such as the working frequency, data transfer rate, modulations and water temperature. The results show that some combinations of temperature and working frequency generate better results than others.

The tenth paper, entitled "Hybrid MANET-DTN and a new algorithm for relay nodes selection" by Papaj et al., proposes a new approach to a selection of the secure relay nodes in hybrid MANET networks. The proposed algorithm enables to select the relay nodes, that will come into contact with other mobile nodes located in different network areas with regards to trust and game theory. Authors implemented the designed mechanism into the OPNET modeler simulation environment, and final results of performance analysis are promising.

The eleventh paper, entitled "A Trust-distortion Resistant Trust Management Scheme on Mobile Ad Hoc Networks" by Hosseini et al., proposes a Trust-distortion Resistant Trust Management Scheme (TRTMS) which provides nodes with an accurate 
estimation on other nodes' behavior and enables them to handle different trust-distortion attacks in a multi-attack environment. Simulation results prove that TRTMS significantly outperforms the existing alternatives in the literature in presence of simultaneous and contradictory different trust-distortion attacks.

The twelfth paper, entitled "An Augmented Routing Algorithm for Trusted Detection of Link Failures in MANETs" by Rahul et al., proposes an algorithm using an efficient cryptosystem with Cipher text List Validator (CLV) and a communal auditing scheme for the validation of certificate received from individual nodes. For constructing the framework, the proposed algorithm with five phases has a network setup phase, data routing phase, communal auditing phase, error node detection phase and a data receiver phase. This framework makes the MANET node build a safe routing topology by effectively judging the harmful nodes as well as the unfaithful information accepted from supplementary nodes.

The thirteenth paper, entitled "Node Re-Routing and Congestion Reduction Scheme for Wireless Vehicular Networks" by Fazio et al., focuses on the optimization of traffic flowing in a vehicular environment with vehicle-2-roadside capability. The proposed idea exploits the information that is gathered by road-side units with the main aim of redirecting traffic flows (in terms of vehicles) to less congested roads, with an overall system optimization, also in terms of Carbon Dioxide emissions reduction.

The fourteenth paper, entitled "Visual Hysteresis Based Dynamic Interference Shaping for Real-time Video Services in Dense Deployed Cellular Networks" by Lu et al., presents the visual hysteresis effect-based dynamic interference shaping method in order to assure QoE of real-time video service. A novel QoE prediction model was proposed to predict the QoE for real-time video services, in which the temporal complexity and hysteresis effect of HVS have been investigated. Additionally, the QoE prediction model for best effort (BE) services was presented. The packet loss rate for real-time video services caused by $\mathrm{BE}$ services is analyzed and the rate scaling factor (RSF) is introduced to assure the QoE for real-time video services and BE services. Finally, the authors investigated the utility function for multi-service and utilized particle swarm optimization (PSO) method to obtain the optimal RSF. Simulation results demonstrated that proposed method considerably improved QoE of real-time video service and network performance.

The fifteenth paper, entitled "Analysis of neighbourhood relations for femtocell networks" by Sedlacek et al., presents the analysis of neighbourhood relations in an LTE based network and focus on neighbour relations of densely deployed femtocells. A number of neighbouring cells and their relations have a direct impact on the PCI (Physical Cell Identities) assignment algorithm design. The PCI assignment algorithms can be adapted and can be optimized to the actual state of a network.

The sixteenth paper, entitled "Efficient RFID Authentication Using Elliptic Curve Cryptography for the Internet of Things" by Shen et al., analyzes the security weaknesses found in the Chen et al.'s RFID authentication scheme and proposes a new RFID authentication scheme using Elliptic Curve Cryptography (ECC). Security analysis results show that the proposed scheme can meet security requirements of RFID authentication while requiring no extra cost in terms of performance.

The seventeenth paper, entitled "A Fairness-Enhanced Micropayment Scheme" by Liu et al., presents an enhanced fairness micropayment scheme to prevent the bank from obtaining the illegal benefits and resist against the collusion attack. In addition, considering that most of the users have no motivation to verify the result due to the tiny transaction value, the observers are employed to audit the process for ensuring the fairness. 
The eighteenth paper, entitled "A Countermeasure to SQL Injection Attack for Cloud Environment" by Wu et al., proposes a mechanism called CCSD (Cloud Computing SQLIA Detection) to detect SQLIAs. CCSD does not require any access to the application's source code. Hence, it can be directly applied to existing cloud environments. The experimental results demonstrate that CCSD has high accuracy, low false positive rates and low time consumption.

The nineteenth paper, entitled "Secure Data Access and Sharing Scheme for Cloud Storage" by Li et al., proposes a scheme for cloud storage based on the intractability of the discrete logarithm problem, where the authors utilize the user authentication scheme to deal with the data access problem. According to the analysis, the authorized users can access the rightful resources and verify the validity of the shared data, but cannot transfer the permission to any other party. At the same time, the confidentiality and integrity of data can be guaranteed.

The twentieth paper, entitled "Exploring the Impact of QR Codes in Authentication Protection: A Study Based on PMT and TPB" by Yang et al., contributes to the extant literature by integrating two theories, Protection Motivation Theory (PMT) and the Theory of Planned Behavior (TPB), and measuring them in a new application focus (QR codes for authentication protection); as well as investigating how the collaboration of computers and mobile devices enhances the protection of users' online credentials. The results of the study additionally provide insights for IT developers for future directions in the development of authentication protection.

The twenty-first paper, entitled "Malicious Events Grouping via Behavior Based Darknet Traffic Flow Analysis" by Pang et al., proposes a host behavior based darknet traffic decomposition approach to identifying groups of malicious events from massive historical darknet traffic. The proposed approach segment the traffic from each source host into flows, and filter out the scan flows with their width larger than length. The resulting scan flows are divided into three categories: port scan, IP scan and hybrid scan. In most cases, hard features are sufficient for scan flows grouping for each category.

The twenty-second paper, entitled "An Improved Remote User Authentication Scheme Using Elliptic Curve Cryptography" by Chaudhry et al., analyzes the Huang et al.'s remote user authentication scheme using elliptic curve cryptography, demonstrates that is prone to impersonation/forgery attack and presents an improved scheme to overcome the weaknesses. The proposed scheme is rigorously analyzed informally and formally. Furthermore, a simulation of proposed scheme is carried out by automated formal tool ProVerif. The analysis has shown that proposed scheme is more robust and lightweight as compared with Huang et al.'s scheme. Hence, due to better security and performance, the proposed scheme is more practical.

The twenty-third paper, entitled "Prediction of Speech Quality Based on Resilient Backpropagation Artificial Neural Network" by Orcik et al., presents a system for monitoring and assessment the speech quality in the IP telephony infrastructures using modular probes. The probes are placed at key nodes in the network where aggregating packet loss data. The system dynamically measures speech quality and results are collected on a central server. For data analysis we applied four-state Markov model for modeling the impact of network impairments on speech quality, afterwards, the resilient back propagation (Rprop) algorithm was used to train a neural network.

The twenty-fourth paper, entitled "Priority-aware Packet Pre-Marking for DiffServ Architecture Based on H.264SVC Video Stream Structure" by Przylucki et al., addresses the problem of lack of the Quality of Service (QoS) mechanisms in IP networks developed as a multimedia transmission environment. The growing popularity of Internet multimedia 
services creates new demands on the mechanisms of packet marking, and thus new challenges for the QoS policy. Authors based on the H264SVC codec, hierarchical video streams, and DiffServ architecture. They describe the Weighted Priority Pre-marking algorithm for priority-aware SVC video streaming. The achieved results confirm that video transmission in the DiffServ domain, based on the Weighted Priority Pre-marking packet pre-marking, can provide better perceived video quality than the best effort streaming of multi-layered SVC video.

The twenty-fifth paper, entitled "RePC: A Localization Method Based on Regional Partition and Cooperation in Communication Networks" by $\mathrm{Xu}$ et al., presents a new localization method called RePC to deal with the challenge which is often encountered in most existing localization applications, i.e., irregular network region and heterogeneous nodes decrease the accuracy of most existing localization methods. The authors introduces a robust and energy efficient localization method RePC. Compared with the existing methods, RePC utilizes a simple but energy efficient regional partition algorithm to divide the irregular region into several regular sub-regions. And then RePC introduces a robust sub-regions cooperative algorithm called $\mathrm{ReC}$ to improve the accuracy of the localization method in every sub-region.

The twenty-sixth paper, entitled "The Entanglement Level and the Detection of Quantum Data Transfer Correctness in Short Qutrit Spin Chains" by Sawerwain et al., evaluates a level of entanglement in short qutrit chains. This evaluation is carried out with use of the CCNR criterion and the concurrence measure. Besides, it presents some explicit formulae describing the values of CCNR criterion and concurrence for exemplary short spin chains.

The twenty-seventh paper, entitled "The Optimal Sizing of HSS Database in IMS" by Kellovsky et al., deals with the architecture and standards of IP the Multimedia Subsystem from next generation networks point of view. It describes the structure of the Home Subscriber Server database and then derives equations for sizing of its load in the proposed scenarios. Finally, the authors apply two methods for the database sizing (Erlang $\mathrm{C}$ and Markov chains) and present results of several scenarios.

The twenty-eighth paper, entitled "Waterfall traffic classification: a quick approach to optimizing cascade classifiers" by Foremski et al., is devoted to building a traffic classification system considered as a tool for heterogeneous systems with various IP traffic. The main goal of such a system is to manage the various kinds of networks, e.g. 4G LTE. The system is constructed as a modular and cascade of the Waterfall architecture. Authors widely describe a novel technique for its optimization in terms of CPU time, number of errors, and percentage of unrecognized flows.

Finally, the twenty-ninth paper, entitled "Redesigning the Healthcare Model to Address Obesity Problem Using the Integration of Processes and Mobile Technologies: Facing a Worldwide Epidemic in an Innovative Manner" by Lokshina et al., proposes an effective new healthcare model to prevent obesity in the youth population, incorporating the development of an innovative multi-dimensional cross-disciplinary ICT framework.

This satisfactory selection of papers has been achieved through a successful collaboration. We would like to thank the Editor-in-chief (Professor Ramjee Prasad) and Springer's senior editorial assistants (Meertinus Faber, Anthony Doyle and Jenilyn Jaos) for their support and help in realizing this special issue. We also would like to give our sincere thanks to all the authors for their contributions and the reviewers for contributing with their professional comments to improve selected papers. Special thanks to the organizers of KTTO 2015 (especially to Prof. Miroslav Voznak) and CN 2015 (especially 
to Prof. Piotr Gaj) conferences, for gathering the best papers for this special issue. We hope you will enjoy reading this special issue.

Coordinating Guest Editor: Homero Toral-Cruz

Guest Editors: Albena D. Mihovska, Piotr Gaj, Debiao He, Julio Cesar Ramírez-Pacheco, Miroslav Voznak, Izabella Lokshina

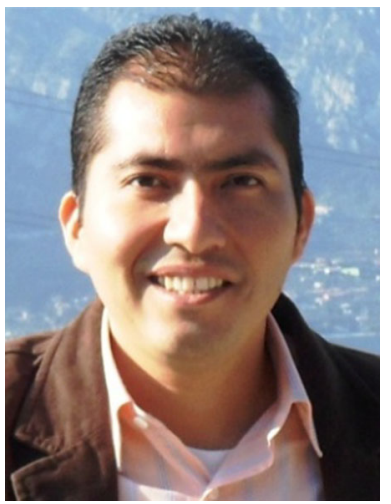

Homero Toral-Cruz received his Ph.D. and MS degrees in Electrical Engineering, Telecommunication option from Center for Research and Advanced Studies of the National Polytechnic Institute (CINVESTAV), Jalisco, Mexico, in 2010 and 2006, respectively. He received his B.Sc. degree in Electronic Engineering from "Instituto Tecnológico de la Laguna", Coahuila, Mexico in 2002. He is currently an assistant professor at Sciences and Engineering department at the University of Quintana Roo, Mexico. Prior to holding this position, he served as an assistant researcher at Electrical Engineering department, Telecommunication section in CINVESTAV, Jalisco, Mexico. His research interest includes network measurement, IP technologies, network security and wireless sensor networks. He has served as guest editor of some high quality journals. In addition, he has served as a chair, organizing committee member and technical program committee member for many international conferences and workshops. Dr Toral is also an active reviewer of some renowned international journals. Furthermore, he has been awarded a national recognition as a researcher (SNI level 1) by CONACYT and has been elected as member of the Mexican Academy of Sciences (AMC).

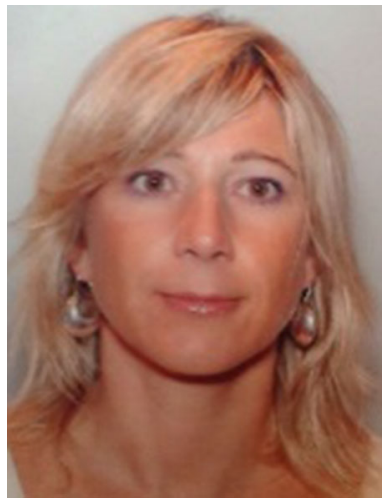

Albena D. Mihovska has a Ph.D. degree in mobile communications from Aalborg University, Aalborg, Denmark, where she is currently an Associate Professor and Head of Standardisation at the Center for TeleInfrastruktur (CTIF), Aalborg University. Currently, she is involved with research related to innovative research concepts for $5 \mathrm{G}$ communication systems, the design and implementation of eHealth services (EU project eWALL) and to optimizing and supporting reliable and high performance intensive data rate communications as required by the Internet of Things. 

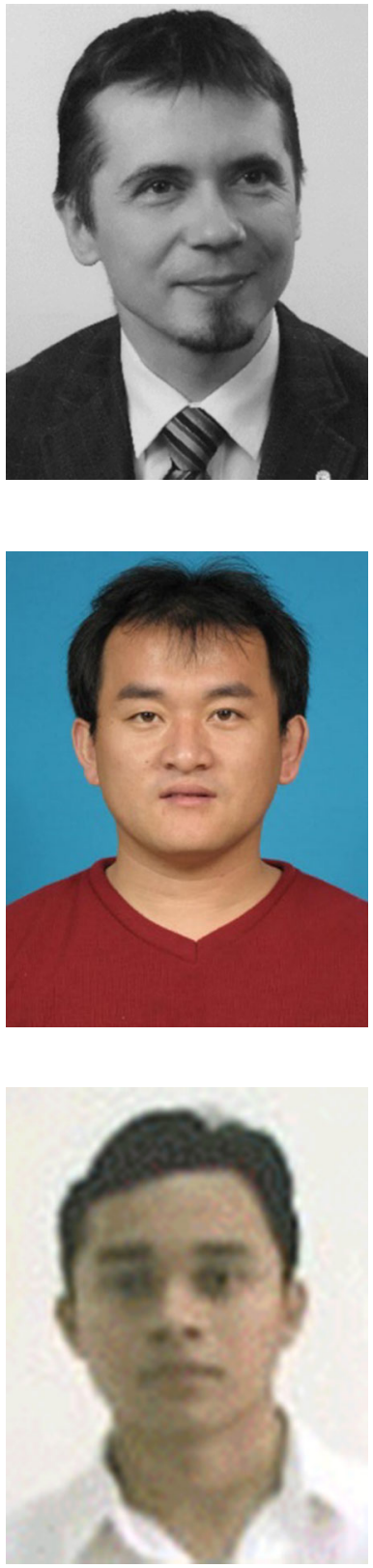

Piotr Gaj (M'10-SM'13) received degrees from the Silesian University of Technology, Gliwice, Poland. He was at a few professional, teaching, and research positions. He authored or coauthored a several dozen papers in the area of industrial systems. He served as a member and reviewer for a few of scientific conferences and journals. His research interests include the area of industrial informatics, including industrial computer networks and systems. He is currently an adjunct in the Department of Automatic Control, Electronic and Computer Science, Silesian University of Technology, Gliwice, Poland. Dr. Gaj is currently the organizing Chair of the 'Computer Networks' international science conference.

Debiao He received his $\mathrm{Ph} . \mathrm{D}$. degree in applied mathematics from School of Mathematics and Statistics, Wuhan University in 2009. He is currently a Professor of the State Key Lab of Software Engineering, Computer School, Wuhan University. His main research interests include cryptography and information security, in particular, cryptographic protocols.

Julio Ramirez-Pacheco (born 1978) is a researcher and full-time professor at Universidad del Caribe, México. He received his Ph.D. in Electrical Engineering at CINVESTAV-IPN, México. His research interests include the analysis and simulation of scale-invariant signals and wavelet entropy analysis of fractal processes. He is a IEEE member and has many publications in the area of wavelet entropy. 

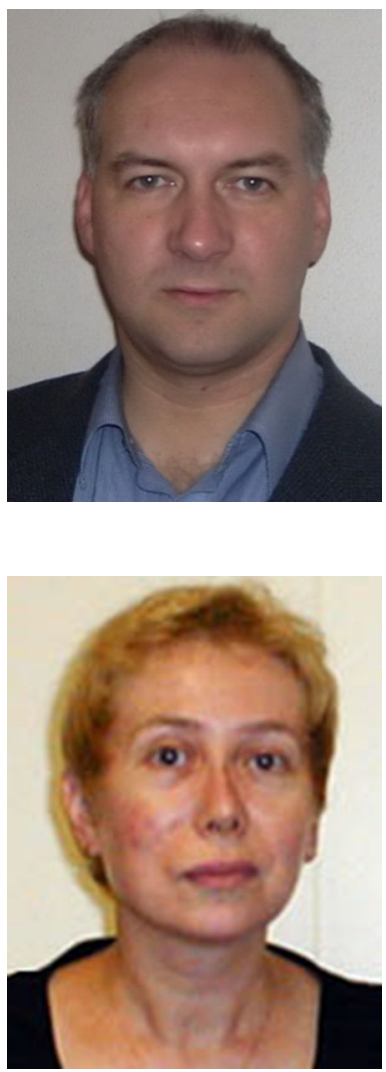

Miroslav Voznak (born in 1971) is a professor with Department of Telecommunications, VSB-Technical University of Ostrava. He received his Ph.D. degree in telecommunications, dissertation thesis "Voice traffic optimization with regard to speech quality in network with VoIP technology" from the VSB-Technical University of Ostrava in 2002. His professional knowledge covers generally Information and Communication technology, in his research, he deals with wireless networks, Voice over IP, service and experience quality, security, big data analytics and optimization problems. He is a senior researcher in IT4Innovations, Czech National Supercomputing Centre in Ostrava and an IEEE Senior Member.

Izabella Lokshina is a Professor of Management Information Systems and Chair of Management, Marketing and Information Systems Department at SUNY Oneonta. She received her Ph.D. in automated control systems (artificial intelligence) from Moscow State Mining University in Moscow, Russia in 1995. Her positions included Senior Scientific Researcher at the Moscow Central Research Institute of Complex Automation and an Associate Professor of Automated Control Systems at Moscow State Mining University. Her main research interests are complex system modeling (communications networks and queuing systems) and artificial intelligence (fuzzy systems and neural networks). She is the author of more than one hundred peer-reviewed publications and she is a recipient of several scholarly awards. Most recently, she has been recognized as 2016 Distinguished Scholar at SUNY Oneonta. 